\title{
Unraveling the chemical history of the Solar System as recorded in extraterrestrial organic matter
}

\author{
George D. Cody ${ }^{1}$, Conel M. O'D. Alexander ${ }^{2}$, A. L. David Kilcoyne ${ }^{3}$, \\ and Hikaru Yabuta ${ }^{1}$ \\ ${ }^{1} \mathrm{GL}$, Carnegie Institution of Washington, \\ 5251 Broad Branch, Washington DC 20015, USA \\ email: g.cody@gl.ciw.edu, hyabuta@gl.ciw.edu \\ ${ }^{2}$ DTM, Carnegie Institution of Washington, \\ 5241 Broad Branch, Washington DC 20015, USA \\ email: alexande@dtm.ciw.edu \\ ${ }^{3}$ Advanced Light Sorce, \\ Lawrence Berkeley Laboratory, Berkeley CA, USA \\ email: alkilkoyne@lbl.gov
}

\begin{abstract}
We have initiated an extensive program of molecular analysis of extraterrestrial organic matter isolated from a broad range of meteorites (spanning multiple classes, groups, and petrologic types), including recent molecular spectroscopic analyses of the organic matter in the Comet $81 \mathrm{P} /$ Wild 2 samples. The results of these analyses clearly reveal the signature of multiple reaction pathways that transformed extraterrestrial organic matter away from its primitive roots. The most significant molecular transformation occurred in the post-accretionary phase of the parent body. However, each of the various chemical transformation trajectories point unambiguously back to a common primitive origin. Applying a wide range of spectroscopic techniques we find that the primitive organic precursor is striking in its chemical complexity exhibiting a broad array of oxygen- and nitrogen-bearing functional groups. The $\pi$-bonded carbon exists as predominately highly substituted single ring aromatics, there exists no evidence for abundant, large, polycyclic aromatic hydrocarbons (PAHs). We find that the molecular structure of primitive extraterrestrial organics is consistent with synthesis from small reactive molecules, e. g. formaldehyde, whose random condensation and subsequent rearrangement chemistry at low temperatures leads to a highly cross-linked macromolecule.
\end{abstract}

Keywords. Comets, asteroids, Solar System, molecular data, ISM: dust

\section{Introduction}

The organic matter in carbonaceous chondrites potentially records a succession of chemical histories that started with reactions in the interstellar medium, followed by reactions that accompanied the formation and evolution of the early solar nebula, and, ultimately, ended with reactions driven by hydrothermal alteration in the meteorite parent bodies. One of the challenges in meteorite research is establishing whether one can identify the chemical signatures of these reactions in the organic fractions and whether any relationship exists between the molecular structure(s) of organic matter within a given meteorite group and the degree of parent body alteration.

There currently exists considerable information regarding the inventory of soluble organic matter derived from numerous studies across a number of carbonaceous chondritic 
groups (e.g., reviews by Hayes 1967, Cronin et al. 1988, Botta \& Bada 2002, Sephton $2002)$. It is well known that the vast proportion $(>70 \%)$ of organic matter in carbonaceous chondrites exists as an insoluble, perhaps macromolecular, solid; defined here as Insoluble Organic Matter (IOM) (Hayes 1967, Cronin et al. 1988). Over the past three decades, substantial progress has been made at characterizing IOM using various analytical techniques. For example, pyrolysis gas chromatography (pyr-GCMS) has been used extensively as a means of characterizing the IOM fraction (Hayatsu \& Anders 1981, Sephton \& Gilmour 2002, Kitajima et al. 2002).

The analysis of various carbonaceous chondrites revealed a predominance of single ring aromatic compounds with a range of alkyl substituents, alkylated phenols, and naphthalenes and alkyl naphthalenes (Hayatsu \& Anders 1981, Sephton \& Gilmour 2002). More recently, pyr-GCMS data were used to characterize the effects of thermal metamorphism on a suite of CM chondrites (Kitajima et al. 2002). Pyrolysis GCMS is an excellent tool for this purpose as these analyses are reproducible and reliably reflect differences in chemical structure among different macromolecules. This stated, it is not always a simple task to reconstruct an accurate structure of a given macromolecule from the molecular constituents in the pyrolysate. This is because considerable chemical modification can occur during the pyrolytic liberation of molecules. There is also the issue of disproportionation, wherein the pyrolysate maybe chemically very different from the non-pyrolyzable residue.

Solid-state nuclear magnetic resonance (NMR) spectroscopy has been shown to be a highly effective analytical tool for establishing the types and distributions of organic functional groups in amorphous organic solids. While lacking the molecular detail of pyr-GCMS, solid-state ${ }^{13} \mathrm{C}$ NMR can provide a quantitative, albeit averaged, structural picture of organic macromolecules. Solid State ${ }^{13} \mathrm{C}$ NMR was first applied to meteoritic IOM by Cronin et al. (1987) who analyzed partially demineralized IOM fractions of Orgueil (CI1), Murchison (CM2), and Allende (CV3). Later, Gardinier et al. (2000) revisited the analysis of Murchison (CM2) and Orgueil (CI1) IOM with Solid State ${ }^{13} \mathrm{C}$ NMR. In 2002, Cody et al. provided a comprehensive analysis of Murchison employing both ${ }^{1} \mathrm{H}$ and ${ }^{13} \mathrm{C}$ solid state NMR experiments. In particular, they performed several independent, yet complementary, experiments to better refine the structural picture of Murchison's IOM fraction.

An example of the solid-state ${ }^{13} \mathrm{C}$ NMR spectrum of Murchison (CM2) IOM is presented in Figure 1. The likely presence of various organic functional groups is clearly evident, where the most abundant functional group is likely aromatic carbon (at $\sim 129 \mathrm{ppm}$ ). It is important to note that the source of the line broadening is not due to any resolution limit of solid state NMR. Rather, the broad lines reflect considerable chemical complexity, i. e., any given functional group exists in a multiplicity of local electron environments. Cody et al. (2002) developed a self-consistent, albeit statistical, picture of the organic structure of Murchison's IOM. What is concluded is that the IOM structure is dominated by small highly substituted aromatics, highly branched and oxygen substituted aliphatics, and abundant carbonyl and carboxyl moieties. How such a structure could have evolved and where the most likely environment was for its synthesis is not possible to determine from the study of a single meteorite.

\section{Low temperature alteration of IOM}

Cody \& Alexander (2005) published an extensive NMR study of IOM from four type 1 and 2 chondrites spanning multiple groups (CR, CI, CM, and Tagish Lake an ungrouped $\mathrm{C} 2$ ). What was observed was a spectacular range in organic structure where the apparent 


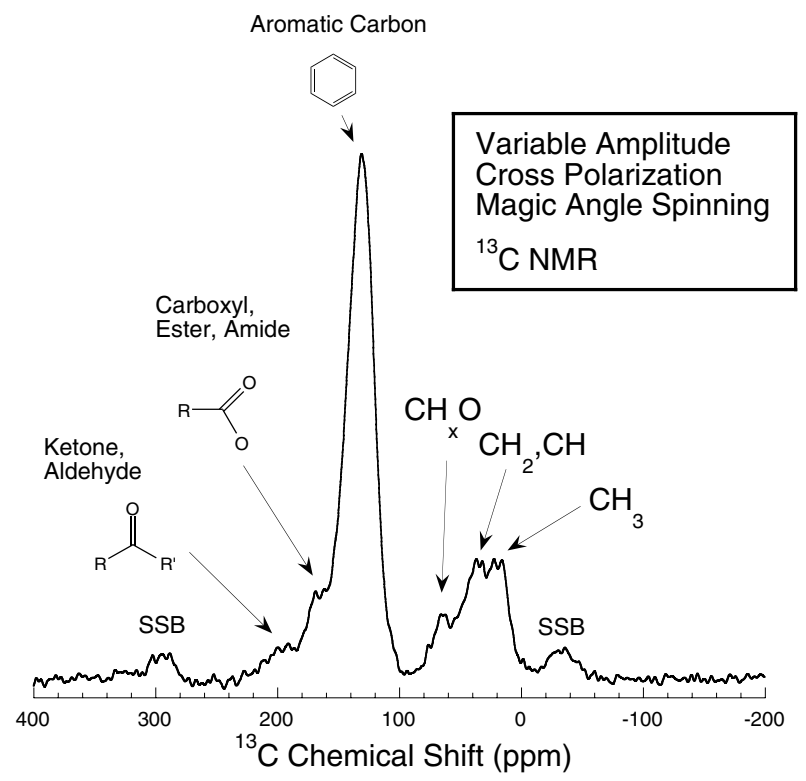

Figure 1. The Solid-state ${ }^{13} \mathrm{C}$ NMR spectrum of Murchison IOM. The various spectral features are labeled with the most likely organic functional groups. Note that the small peaks labeled SSB, correspond to spinning side-bands derived from the most intense peak at a frequency of $\sim 129 \mathrm{ppm}$. The chemical shift is recorded as the parts per million shift relative to the ${ }^{13} \mathrm{C}$ frequency $(\sim 75 \mathrm{MHz}$ at $7.05 \mathrm{~T})$. The shift is normalized such that the frequency of methyl groups in tetramethyl silane are defined as having a shift of $0 \mathrm{ppm}$.

aromatic carbon abundance rose from a low of $\sim 48 \%$ (for the CR) up to $\sim 80 \%$ in the case of Tagish Lake (Figure 2). Remarkably none of the type 1 or 2 chondrites have ever experienced heating in excess of $\sim 100{ }^{\circ} \mathrm{C}$ during parent body alteration. This raises the question as to what type of chemistry could lead to such a remarkable difference in molecular structure, assuming that all IOM has a common precursor.

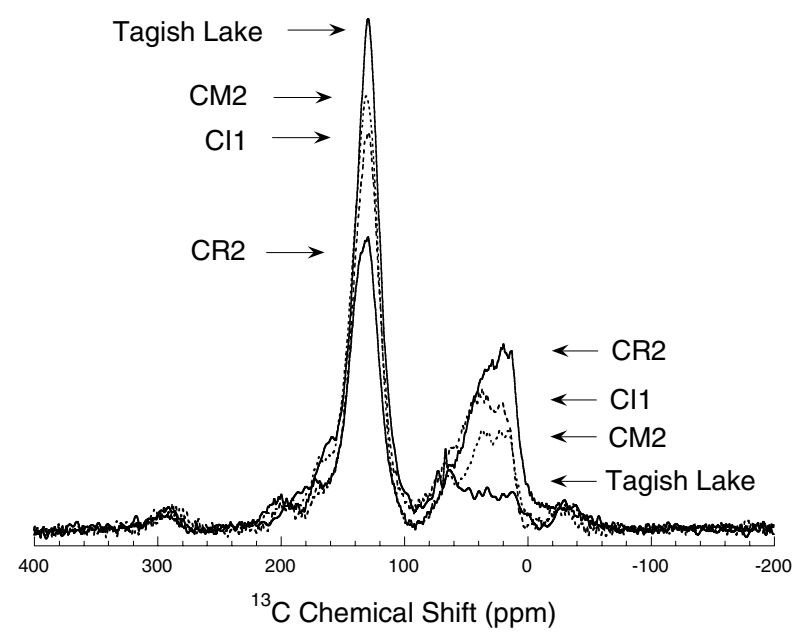

Figure 2. An over-lay of the solid state NMR spectra of IOM from the CR, CI, CM, and Tagish Lake groups of carbonaceous chondrites. Note the tremendous range in apparent aromatic carbon content (at $\sim 129 \mathrm{ppm})$. 
Through a series of independent NMR experiments Cody \& Alexander (2005) proved that the significant differences in the chemistry of IOM across the four meteorite groups (Figure 2) reflect primarily differences in the abundance and types of $s p^{3}$ bonded carbon. The most likely interpretation for this chemical variation is that the differences in type 1 and 2 IOM record a progressive trend in chemical oxidation that would require the presence of a soluble oxidant in the aqueous phase during parent body alteration. One possibility is hydrogen peroxide, a likely constituent in the icy mantles of dust grains subjected to UV photolysis. In this study of meteoritic IOM, therefore, the CR2 is apparently the least and Tagish Lake is apparently the most chemically degraded. The selective enrichment in aromatic moieties and nano-diamond suggested that these constituents of IOM are relative inert to this oxidation and that their relative abundance may provide a measure of the extent of oxidation. The organic matter contained within the matrices of type 1 and 2 carbonaceous chondrites, therefore, may provide a unique record of the oxidative nature of aqueous fluids that altered each meteorite's parent body.

\section{The effect of thermal metamorphism on IOM}

In order to study the organic structure of the thermally metamorphosed type 3 chondrites, one is faced with the problem that there is generally much less organic matter and we can rarely obtain sufficient amounts of IOM to acquire solid state NMR spectra. To circumvent this difficulty we apply micro Carbon X-ray Absorption Near Edge Structure (C-XANES) spectroscopy. C-XANES provides similar information to that of solid state NMR. While C-XANES lacks the ease of quantification that solid state NMR provides, samples as small as a few microns in diameter can easily be analyzed.

Through the analysis of $\sim 25$ different IOM samples obtained from type $3 \mathrm{CV}, \mathrm{CO}$, and Ordinary chondrites, a clear picture of the effect of thermal metamorphism on IOM has been obtained (Cody et al., submitted). Prolonged exposure to elevated temperatures $\left(T>200{ }^{\circ} \mathrm{C}\right)$ in the chondritic parent body progressively transforms primitive IOM into a highly aromatic material that exhibits semi-conductor-like electronic properties. In addition to showing a clear correlation between IOM structure and mineralogic indicators of metamorphic grade, Cody et al. (submitted) also showed that laboratory heating of primitive IOM (e.g., from Murchison) leads to the formation of organic structures that are identical to that in heated chondrites. These laboratory derived transformation kinetics can be used as a cosmothermometer revealing that the organic matter spanning the type 3 to type 4 chondrites records a temperature range up to $950{ }^{\circ} \mathrm{C}$.

Finally, a significant result of the study of the type 3 chondrites is the unambiguous demonstration that the constituent primitive organic matter in type 1 and 2 chondrites may be thermally transformed into a structural state that is nearly identical to that observed in thermally metamorphosed type 3 chondrites. As it is generally agreed that type 1 and 2 chondrites never experienced temperatures much in excess of $100{ }^{\circ} \mathrm{C}$; these results require that, in general, meteoritic organic matter was synthesized cold and, at least in the case of the type 1 and 2 chondrite parent bodies, accreted cold.

\section{Comparison with comet $81 \mathrm{P} / \mathrm{Wild} 2$ organics}

The Stardust comet sample return mission from Comet $81 \mathrm{P} /$ Wild 2 provides an unprecedented opportunity to assess the organic chemistry of what may be the most primitive Solar System material, providing a link to the molecular cloud material that was the ultimate source of the matter from which our Solar System originated. It is immediately compelling to see whether any chemical connection exists between the cometary organic 
matter and meteoritic IOM. Only very small particles were collected by necessity and, therefore, C-XANES provides a robust method for organic structure analysis. Combining $\mathrm{C}-, \mathrm{N}-$, and O-XANES also allows for the robust determination of elemental ratios, e. g. $\mathrm{O} / \mathrm{C}$ and $\mathrm{N} / \mathrm{C}$.

To date, eight organic cometary particles analyzed have been using C-XANES (Sandford et al. 2006, Cody et al. 2008). In Figure 3b, the elemental chemistry of these particles are shown along with a single measure of an interplanetary dust particle, as well as a representative group of chondritic IOM (Alexander et al. 2007). In general, the comet organics are enriched in both oxygen and nitrogen relative to meteoritic IOM. The CXANES spectra reveal what appears to be a chemical evolutionary trend moving from the most oxygen-rich cometary organics (Comet A) through less oxygen-rich organics (Comet B) to Murchison IOM (Figure 3a).

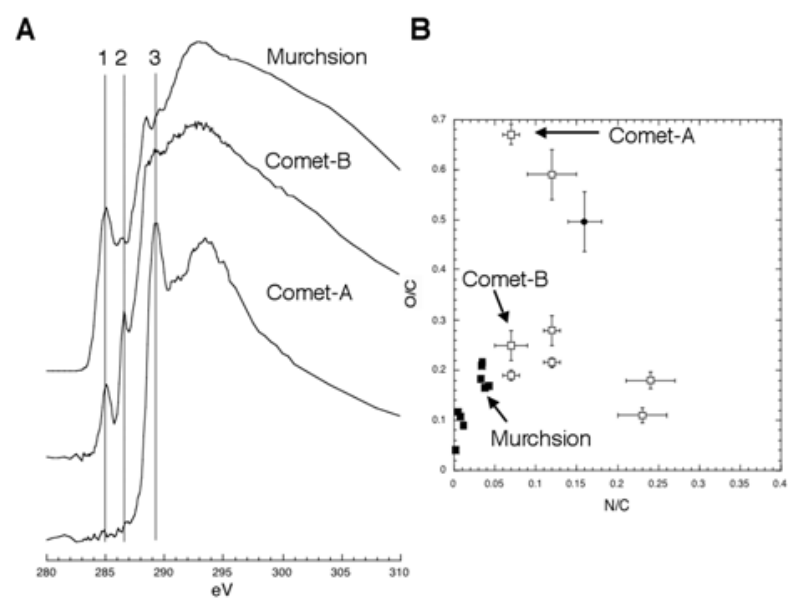

Figure 3. A) Carbon X-ray Absorption Near Edge Structure Spectra of two comet particles compared with IOM isolated from the Murchison (CM2) chondrite. Peak 1 corresponds to $s p^{2}$ bonded carbon, i. e., either aromatic or olefinic; Peak 2, corresponds to a keto-substituted olefin and is characteristic of the degradation of polyol (e.g. sugars). Peak 3 highlights the presence of $s p^{3}$ carbon bonded to oxygen, e. g. alcohol or ether. B) particle "Comet-A" is very oxygen-rich with chemistry dominated by alcohol and/or ether; particle "Comet-B" has an elemental composition not far from chondritic IOM; the elemental composition of Murchison IOM still contains appreciable oxygen and nitrogen. The loss of oxygen moving from particle "Comet-A" to Murchison is consistent with the progressive elimination of OH groups from sugar-like molecules yielding keto, carboxyl, and olefinic functional groups.

Any chemical pathway connecting the most oxygen-rich cometary organic matter to the molecular matter that is chondritic IOM requires the formation of considerable $s p^{2}$ bonded carbon (e.g., peak 1, Figure 3a) at the expense of oxygen-bearing $s p^{3}$ carbon (e.g., Peak 3, Figure 3a). Such a transformation would have to occur at very low temperatures, even type 2 chondritic IOM never experienced temperatures in excess of $100{ }^{\circ} \mathrm{C}$. The apparent trend in chemical evolution observed in Figure $3 \mathrm{a}$ is potentially informative. In particular, the growth of the peak at $286.5 \mathrm{eV}$ (Peak 2, Figure 3a) is consistent with the transformation of complex sugar-like molecules into unsaturated keto-bearing structures through the elimination of $\mathrm{OH}$. Support for this pathway is provided by the observation of the progressive growth of a peak at $286.5 \mathrm{eV}$ resulting from secondary electron damage of polysaccharides (Cody 2000). 


\section{A self-consistent picture of IOM's origins and subsequent evolution}

It appears likely that both cometary and meteoritic organic matter are linked through a common molecular precursor. The most likely candidate would be complex sugars. The source of such sugars and sugar derivatives would be through the formose condensation of formaldehyde. Subsequent evolution involving Aldol, retro-Aldol, elimination, Amidori rearrangements, and Diels-Alder cylcoaddition reactions would naturally lead from a complex mixture of sugar molecules to the highly complex organic structure observed in type 1 and 2 chondritic meteorites. Subsequent heating in the parent bodies converts this primitive IOM into the highly conjugated aromatic structures identified in type 3 chondrites. Extraterrestrial organic matter in meteorites, therefore, traces its origins back to cold chemistry from which one of the most abundant carbon molecules in the Galaxy, formaldehyde, was formed. Whereas the meteoritic organic history is simplified with such a scenario, the presence of relatively large sugar-like molecules and partially modified sugar-like molecules in comets is interesting. Presumably such chemistry would require liquid conditions for at least some short period in the comet's history. It is evident from the on-going organic and inorganic analyses of the Comet $81 \mathrm{P} /$ Wild 2 samples that we have much more to learn about comets.

\section{Acknowledgements}

We gratefully acknowledge financial support from NASA's Origins of the Solar Systems Program, Stardust Data Analysis Program, and NASA Astrobiology. C-XANES analyses were performed at the Advanced Light Source, a US-DOE supported facility. We are deeply grateful for the help and guidance provided by Dr. Tohru Araki.

\section{References}

Alexander, C. M. O'D., Fogel, M., Yabuta, H., \& Cody, G. D. 2007, Geochim. Cosmochim. Acta, 71,4380

Botta, O. \& Bada, J. L. 2002, Surv. Geophys., 23, 411

Cody, G. D. 2000, in: W. Meyer-Ilse, T. Warwick, \& D. Attwood (eds.), X-ray Microscopy: Proceedings of the Sixth International Conference, (American Institute of Physics, Melville, NY)

Cody, G. D. \& Alexander, C. M. O'D. 2005, Geochim. Cosmochim. Acta, 69, 1085

Cody, G. D., Alexander C. M. O'D, \& Tera F. 2002 Geochim. Cosmochim. Acta, 66, 1851

Cody, G. D., et al. 2008, Meteorit. Planet. Sci., In Press

Cody, G. D., et al., Earth Planet. Sci. Lett., submitted

Cronin, J. R., Pizzarello, S., \& Cruikshank, D. P. 1988, in: J. F. Kerridge \& M. S. Mathews (eds.), Meteorites and the Early Solar System, (Tucson: University of Arizona Press), p. 819

Cronin, J. R., Pizzarello, S., \& Frye, J. S. 1987, Geochim. Cosmochim. Acta, 51, 299

Gardinier, A., Derenne, S., Robert, F., Behar, F., Largeau, C., \& Maquet, J. 2000, Earth Planet. Sci. Lett. 184, 9

Hayes, J. M. 1967, Geochim. Cosmochim. Acta, 31, 1395

Hayatsu, R. \& Anders, E. 1981, Topics. Curr. Chem., 99, 1

Kitajima, F., Nakamura, T., Taraoka, N., \& Murea, T. 2002, Geochim. Cosmochim. Acta, 66, 163

Sandford, S. A., et al. 2006, Science, 314, 1720

Sephton, M. A. 2002, Natural Product Reports, 19, 292

Sephton, M. A. \& Gilmour, I. 2002, ApJ, 540, 588 


\section{Discussion}

Pendleton: I was hoping to hear you respond back towards George Flynn who said about the IOM, and the fact that we could actually have been fooled all along by creating that aliphatic structure that seems to have matched in the 3.4 micron regions so well to the ISM. However, Lou Allamandolla and I did notice some time ago that at the longer wavelengths Murchison and the ISM do not match because Murchison seemed to have more oxygen.

CoDy: I did not want to go too far into the details here. I could show you how one could create all the chemistry exhibited in IOM from a combination of amidori rearrangements, elemination, tautoterimization, etc. that will give you a certain amount of methyl carbon. We know precisely how much methyl, methylene, and methine we have, how much is oxygen substituted and so forth. So we can calculate the IR spectrum and it will look just like what you see. The thing that intrigues me is that this similarity suggests a common precursor. What I have been thinking based on what I have learned at this meeting is that we see this wonderful proposed structure that you and Lou Allamandolla put together that accommodates two features that are clearly observed, on the one hand there is the interesting aliphatic structure, on the other hand there are the large PAHs. This suggests to me that we really have two chemical systems represented here. The very large PAHs seem to be part of a different chemical pathway that is not observed in the IOM fraction. IOM looks like pure formaldehyde chemistry. PAH's likely form from a different pathway. Combine formaldehyde derived IOM with large PAH's and you may get an IR spectrum that matches what you observe.

HenNing: You talked about the nitrogen content of your sample, so can you tell us a little bit more where the nitrogen is? Is it in the nanodiamond or is it somewhere else?

CoDY: There is nitrogen in nanodiamonds which is why we can observe them with NMR. I have never detected nanodiamond using STXM, which has a spatial resolution of 30 to $40 \mathrm{~nm}$ insufficient to resolve nanodiamonds. Most of the nitrogen in IOM is amine, there is very little nitrile or imine. We detect these, but the concentration is less than $5 \%$ of the total nitrogen in all of the IOM we have studied and also in George Flynn's IDP's. The spectra are almost identical, you observe the same nitrogen chemistry over and over again.

ZIURYS: I like this idea of starting with formaldehyde and methanol and glycoaldehyde. Formaldehyde and methanol of course are really abundant interstellar molecules and glycoaldehyde has been seen in the galactic centre. So this seems to be at least the beginnings of the gas phase formose reaction that produces at least 2-carbon sugar and those could be the interstellar starting materials for what you're talking about, so I like it!

CoDY: Thank you. The tricky thing is how do you keep the chemistry going beyond glycoaldehyde and where is the appropriate environment for this chemistry. In order to have $25 \%$ furanic species in IOM requires a lot of aldose sugars present.

ZIURYS: How sure are you that it is just this particular furan?

CoDy: Everything that I presented here is an example of molecular constituents in IOM. What we know for a fact is that the chemical complexity in these NMR spectra tell you 
that you are always observing an ensemble of compounds. If the actual chemistry were as simple as the examples I showed you, you would observe a nest of really sharp resonance lines.

SLOAN: My concern about is how we're going to drive the mixture of aromatics and aliphatics. Whatever mechanism does this, it has to happen pretty quick, because we see these unusual spectra in post-AGB objects. You don't have to go to molecular clouds to get this mixed chemistry.

CoDy: I have been listening with great intrigue to the PAH story. I am outside of this field, which is why this meeting has been superb for me. My astronomy colleagues at the Carnegie often ask me why I don't observe a lot of PAH's in IOM and I cannot provide them an answer yet. I also cannot answer your question, one which I agree is profound. What I can say is that if we want to connect IR spectra observed in diffuse molecular clouds and that observed in Murchison IOM, it is not beyond the realm of possibility that there is similar chemistry going on out there that yields products that mimic what we observe in meteoritic IOM.

Mumma: Just a comment on formaldehyde in comets. The situation is that the monomer of formaldehyde is typically on the order of half percent relative to water, so there's lot of that. But in Halley, for example, the polymer of formaldehyde would be about $7 \%$, and in Hale Bopp it is about $12 \%$ relative to water. So you can imagine these icy mantle grains with that composition falling in the nebula and some vaporizing near the asteroid region and forming later into the IOM. You might have some really profound source of material you are talking about here. You could be dead on.

CoDy: I agree. Thinking about polymerization theory, we can begin to ask how much of the carbon that we observe as IOM is related to the abundance of primitive chemistry out there. In other words how efficient could the IOM forming chemistry be. Are we looking at sub percent, 5 , or $10 \%$ polymerization? I should note that one thing I did not emphasize in this talk is that IOM is very tough in regards to temperature. If IOM resides in hot regions of the solar nebula, high oxygen or hydrogen abundance will chemically erode it away. If the oxygen and hydrogen content are low, IIOM will hang around even to very high temperatures. I never would have expected this. 